\title{
Hot Electron Modelling of HEMTs
}

\author{
ERIC A. B. COLE ${ }^{\mathrm{a}, *}$, CHRISTOPHER M. SNOWDEN $^{\mathrm{b}, \dagger}$ and SHAHZAD HUSSAIN ${ }^{\mathrm{b}, \ddagger}$ \\ ${ }^{\mathrm{a}}$ Department of Applied Mathematics, ${ }^{\mathrm{b}}$ School of Electronic and Electrical Engineering, \\ University of Leeds, Leeds LS2 9JT, UK
}

\begin{abstract}
The hot-electron two-dimensional HEMT with recessed gate is modelled by solving the Poisson, current continuity and energy transport equations consistently with the Schrödinger equation using a finite difference scheme. New expressions are used for the energy densities inside and outside the quantum wells. A method is described for pinning the conduction band at the contact edge to produce an extremely stable numerical solution. Results are presented for an eight layer GaAs-AlGaAs-InGaAs device.
\end{abstract}

Keywords: HEMT; Schrödinger; Poisson; Current-continuity; Hot electron

\section{INTRODUCTION}

Considerable problems are encountered in the mathematical and numerical modelling of recessed gate High Electron Mobility Transistors (HEMTs) in which hot electrons are considered. Even when the most exacting initial conditions are used, it is often difficult to get the quantum well structure established at the outset for the simulation to continue in a robust fashion. Even without a recess, there is difficulty in pinning the conduction band high enough on the free surfaces of the edge containing the contacts. The problem is made even more difficult with the recess in place because the well structure is compressed into the device. Many attempts have been made to obtain rapid and accurate solutions for these and similar devices
[1-14]. A multigrid method has been developed for the solution of the coupled one-dimensional Poisson-Schrödinger equations [1], and applied to the isothermal HEMT model [2]. The current continuity and energy transport equations have been discretised in a consistent manner using the C-function method [3], and it has been shown that the interpolation of the electron temperature between grid points must be done using a new logarithmic mean rather than the arithmetic mean [4].

In this paper we give details of a simulation involving the solution of the coupled Poisson, Schrödinger, current continuity and energy transport equations for hot electrons and show how the steady state system may be solved numerically in a robust fashion using a modified Newton iteration

* Corresponding author. Tel.: 0113233 5117, Fax: 0113233 5090, e-mail: e.a.b.cole@leeds.ac.uk

${ }^{\dagger}$ Tel.: 0113233 2001, Fax: 0113233 2032, e-mail: csnowden@filct.com

‡ Tel.: 0113233 5117, Fax: 0113242 9925, e-mail: shahzad@amsta.leeds.ac.uk 
scheme. The purpose of this paper is to describe how a stable smooth solution may be obtained, not necessarily the most rapid, although it is shown how the runtime may be reduced by introducing techniques which do not compromise the stability. The ideas presented here could then be applied to faster methods, for example to the multigrid method. Results are obtained for the device shown in Figure 1.

The band offset structure illustrated in Figure 2 will depend on the proportion $u$ of $\mathrm{Al}$ and $v$ of In in the layers of $\mathrm{Al}_{u} \mathrm{Ga}_{1-u}$ As and $\mathrm{In}_{v} \mathrm{Ga}_{1-v}$ As respectively. The dependent variables for which a solution is found are the electrostatic potential $\psi$,

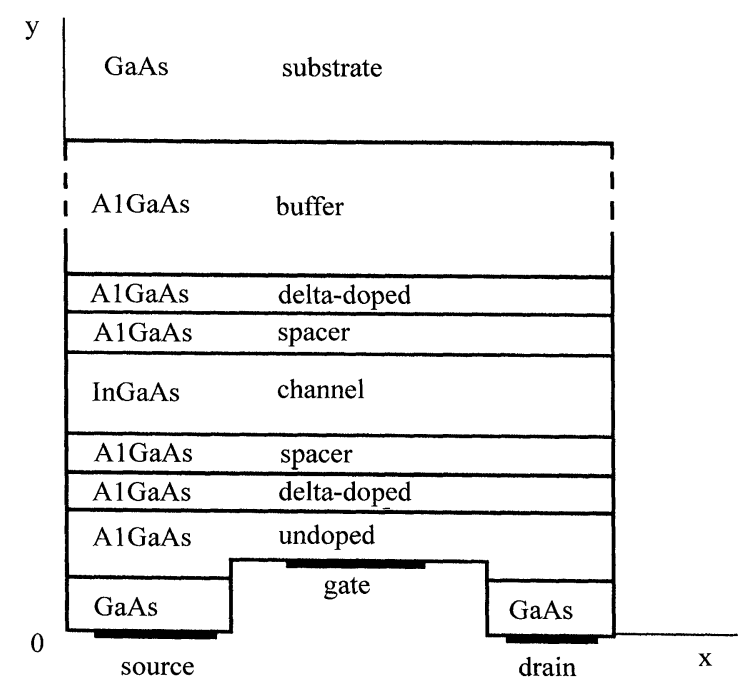

FIGURE 1 Cross-section of an eight-layer GaAs-AlGaAsInGaAs HEMT showing the positions of the recess, contacts and layer structure.
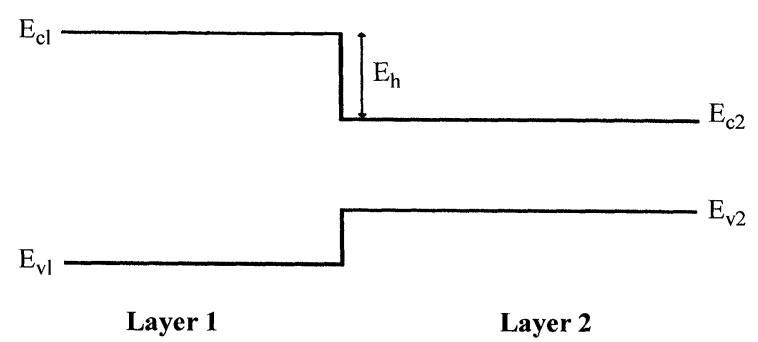

FIGURE 2 The band offset structure between two layers showing the conduction band $E_{c}$ and step $E_{h}$. the electron temperature $T$, and the quasi Fermi level $E_{F}=-q \phi$ where $q$ is the magnitude of the electron charge. The equations to be solved are:

(i) The Poisson equation

$$
\nabla \cdot\left(\varepsilon_{0} \varepsilon_{r} \nabla \psi\right)=-q\left(N_{D}^{+}-n-T^{-}\right)
$$

for the solution of the electrostatic potential $\psi$. Here, $n$ is the total electron density and $\varepsilon_{0}$ is the permittivity of free space. The conduction band edge is then $E_{c}=E_{h}-q \psi$.

(ii) The current continuity equation

$\frac{\partial n}{\partial t}=\frac{1}{q} \nabla \cdot \mathbf{J}, \quad \mathbf{J}=-q \mu n \nabla \phi+\mu k n \nabla T$

for the current density $\mathbf{J}$, where $k$ is Boltzmann's constant. Generally, the mobility $\mu$ will depend on the electric field $\mathbf{E}=-\nabla \psi$.

(iii) The energy transport equation

$\frac{\partial \xi}{\partial t}=q \mathbf{v} \cdot \mathbf{E}-\mathbf{v} \cdot \nabla \xi-\frac{1}{n} \nabla \cdot\left(n \mathbf{v} k_{B} T\right)-\frac{\left(\xi-\xi_{0}\right)}{\tau_{e}}$

where $\xi$ is the average electron energy density, $\xi_{0}$ is the average electron energy evaluated at the lattice temperature $T_{0}$, and $\tau_{e}$ is the electron energy relaxation time depending on $\xi$. It should be noted that the use of the energy balance model and its underlying assumptions may in some circumstances overestimate the velocity overshoot. Specifically, the energy balance model described here neglects convective effects $(\mathbf{v} \cdot \nabla) \mathbf{v}$ and kinetic energy.

(iv) The Schrödinger equation

$$
-\frac{\hbar^{2}}{2} \nabla \cdot\left(\frac{1}{m} \nabla \xi_{i}\right)+\left(V_{x c}+E_{h}-q \psi\right) \xi_{i}=\lambda_{i} \xi_{i}
$$

where $\xi_{i}$ and $\lambda_{i}(i=0,1,2 \ldots)$ are the energy eigenfunctions (normalised) and eigenvalues 
respectively. The forms of the exchange correlation energy $V_{x c}$, effective mass $m$, relative permittivity $\varepsilon_{r}$, conduction band discontinuity $E_{h}, N_{D}^{+}$and $T^{-}$are taken from Adachi [5]. The form of the kinetic energy operator is taken for its hermitian qualities $[15,16]$. Since we are dealing with an essentially two-dimensional problem, the Schrödinger equation must be solved in two dimensions. Because of the slope of $E_{c}$ down towards the drain end of the device (see Fig. 3a). many eigensolutions must be obtained in order to reach energy eigenvalues which intersect with the quantum well towards the source end of the device. Obtaining the required number of eigensolutions is very time consuming. In order to obtain a numerical solution in a realistic time, we assume that the potential is much more slowly varying in the $\mathrm{x}$-direction than the $\mathrm{y}$-direction since the overall device length in the $\mathrm{x}$-direction is of an order of magnitude greater. This enables us to solve the Schrödinger equation in one-dimensional slices perpendicular to the layer structure (that is, in the y-direction). Thus for a given slice, the lowest eigenvalue is obtained immediately rather than having to be built up from many eigensolutions of the full twodimensional model. However, further work must be done to show how this approach can be justified in a more rigorous fashion. We therefore solve the one dimensional

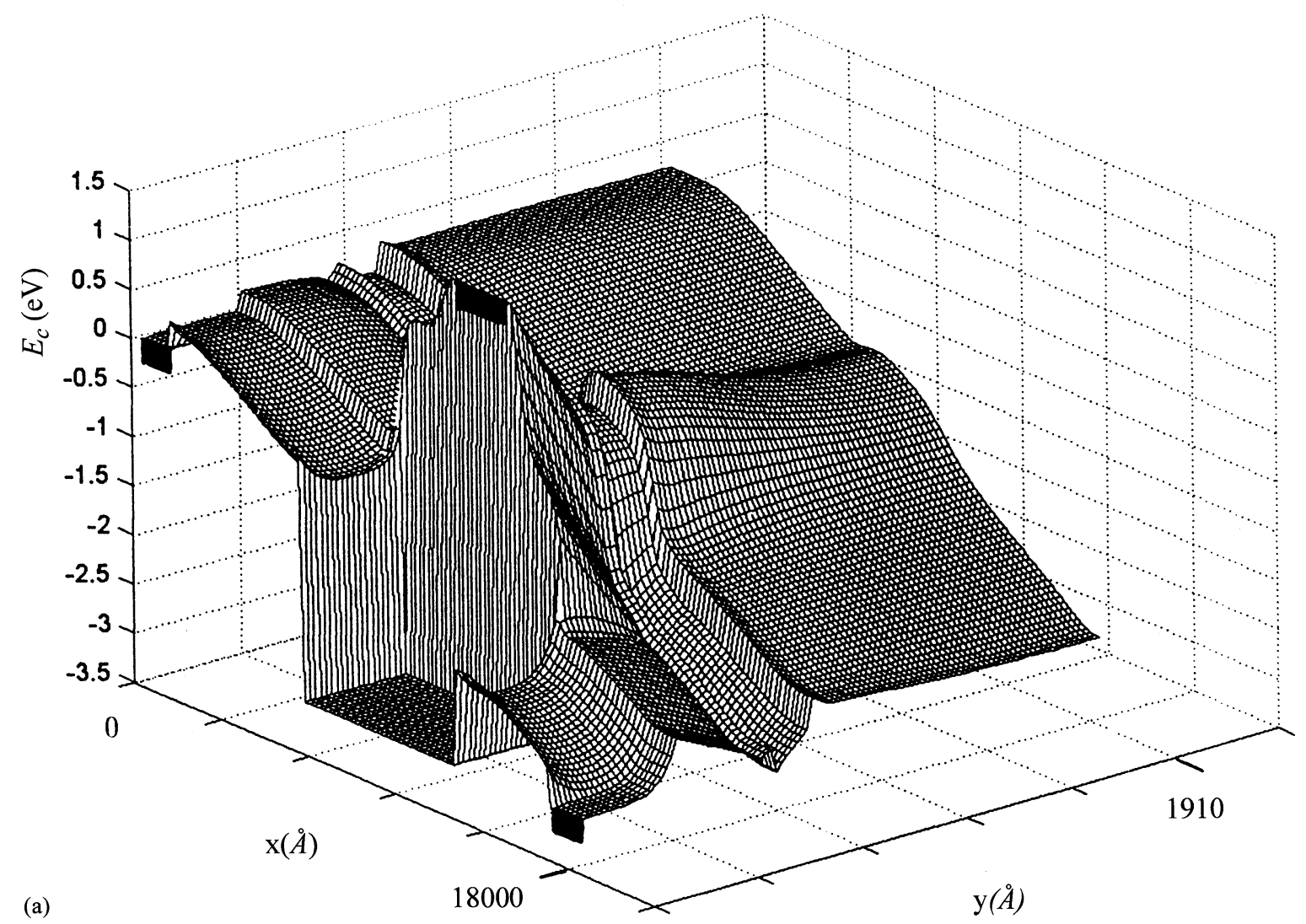

FIGURE 3 Results of the simulation with $V_{g s}=-0.5 \mathrm{~V}$ and $V_{d s}=3.0 \mathrm{~V}$ viewed in the direction of the arrow in Figure 1, with all distances in Angstroms, showing (a) the conduction band $E_{c}$, (b) the electron density $n$, and (c) the electron temperature $T$. 


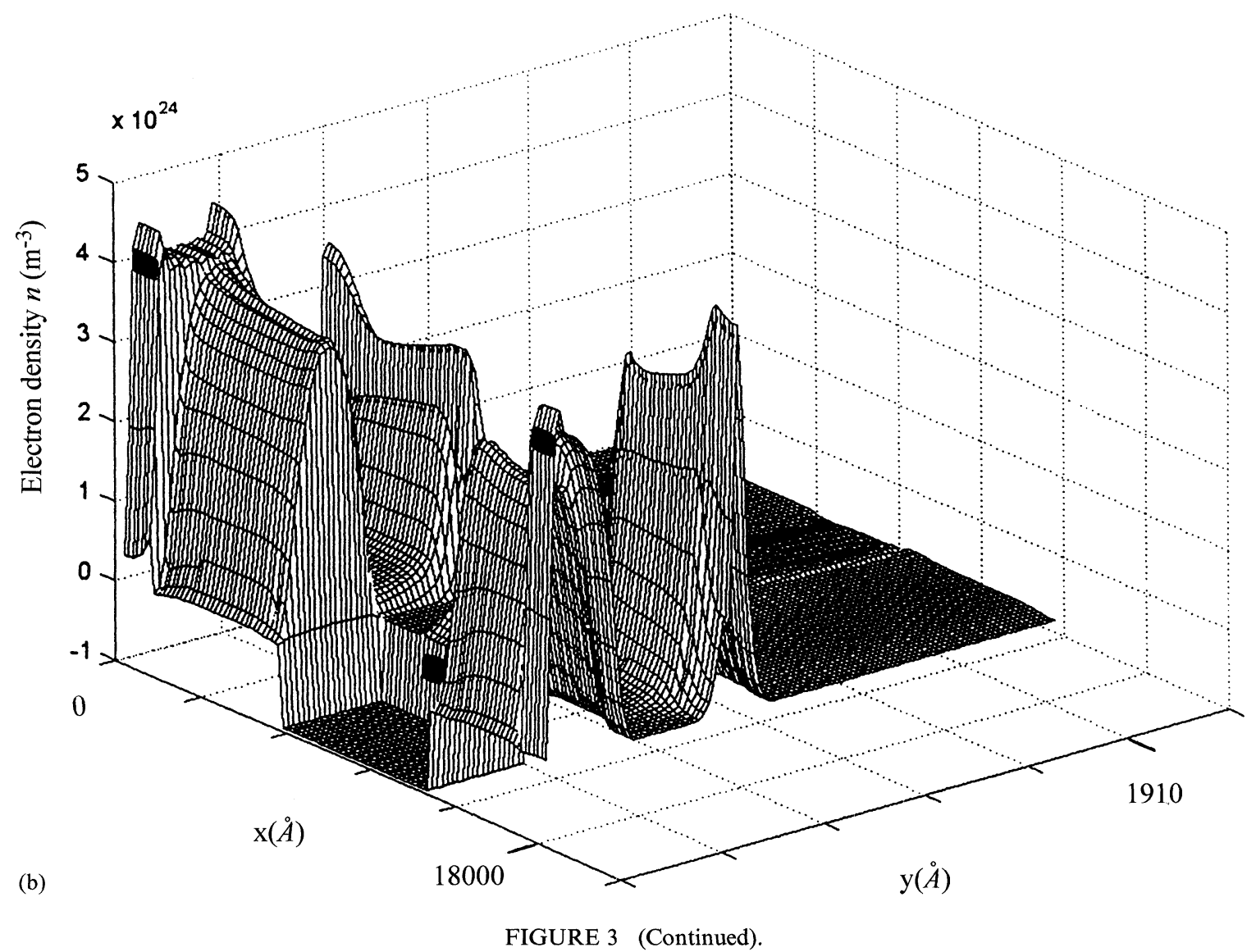

equation

$-\frac{\hbar^{2}}{2} \frac{d}{d y}\left(\frac{1}{m} \frac{d \xi_{i}}{d y}\right)+\left(V_{x c}+E_{h}-q \psi\right) \xi_{i}=\lambda_{i} \xi_{i}$

by imposing the boundary conditions $\xi_{i}(0)=\xi_{i}(Y)=0(i=0,1,2, \ldots)$.

The electron density $n$ inside the quantum wells will be given in terms of the eigensolutions of this equation. The total electron density is given by $n=$ $n_{2}+n_{3}$ where $n_{2}$ is the contribution from the sub-bands given by the solution of the Schrödinger equation and $n_{3}$ is the bulk electron density. We first choose a maximum number $L$ of eigensolutions of the Schrödinger equation with which to work [1]. Outside the potential well defined by
$\lambda_{L-1}<E_{c}$ we have $n=n_{2}+n_{3}$ with

$$
n_{2}=0, \quad n_{3}=N_{c 3} F_{(1 / 2)}\left(\frac{1}{k T}\left(E_{F}-E_{c}\right)\right) .
$$

Inside the well we must avoid the double counting of contributions, and thus we have $n=$ $n_{2}+n_{3}$ with

$$
\begin{aligned}
& n_{2}=N_{c 2} \sum_{i=0}^{L-1}\left|\xi_{i}(y)\right|^{2} \ln \left(1+e^{(1 / k T)\left(E_{F}-\lambda_{i}\right)}\right) \\
& n_{3}=\frac{1}{2} \pi\left(\frac{8 m}{h^{2}}\right)^{3 / 2} \int_{\lambda_{L-1}}^{\infty} \frac{\left(E-E_{c}\right)^{1 / 2}}{1+\exp \left((1 / k T)\left(E-E_{F}\right)\right)} d E
\end{aligned}
$$

In the above expressions, we have $N_{c 2} \equiv(4 \pi m k T)$ $h^{2}$ ) and $N_{c 3} \equiv 2\left(2 \pi m k T / h^{2}\right)^{1.5}$. The expressions for 


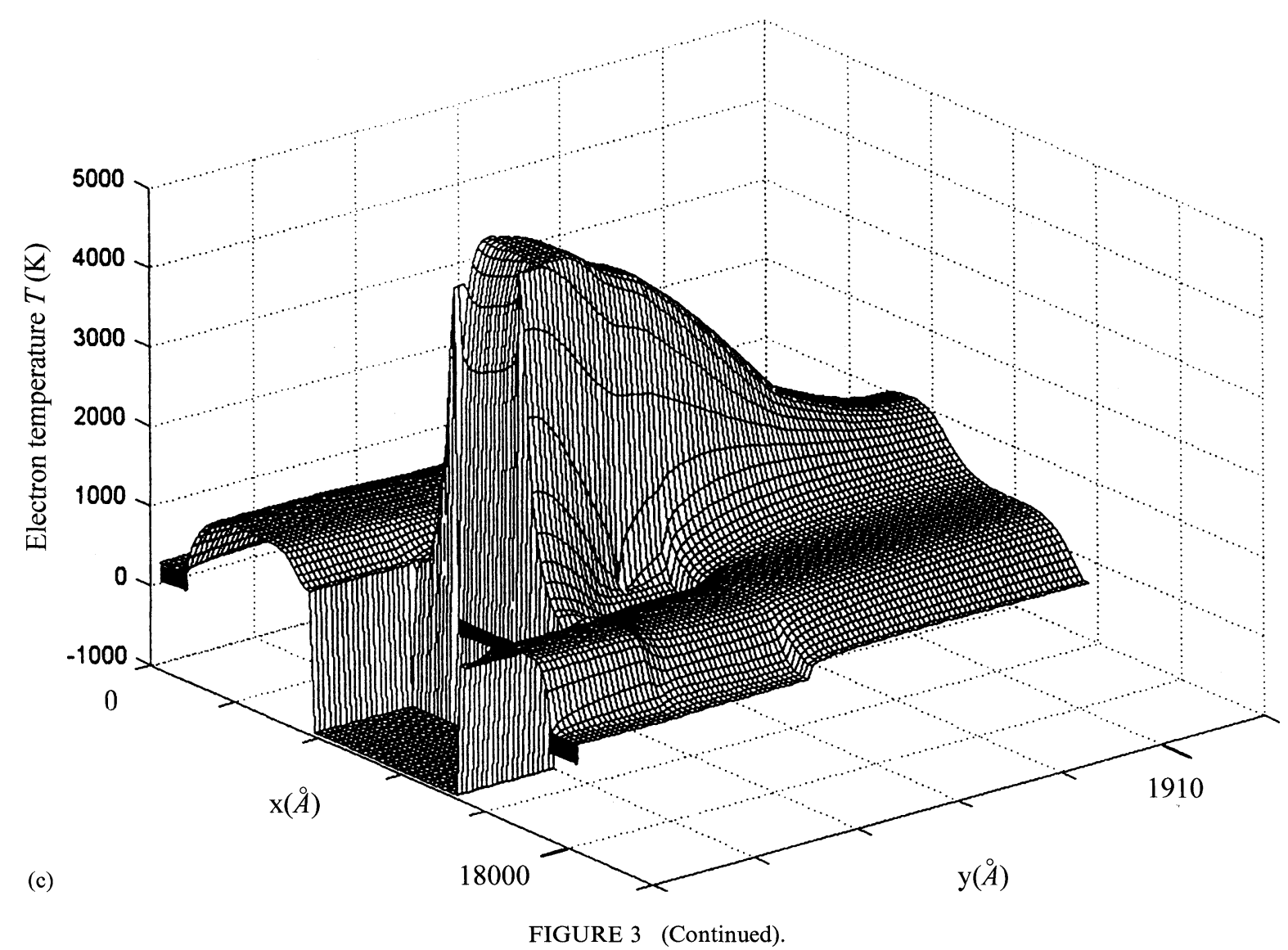

the total energy density $W=n \xi$ will take similar forms. By performing integrals involving the density of states, it can be verified that outside the wells,

$$
W_{2}=0, \quad W_{3}=W_{c 3} F_{(3 / 2)}\left(\frac{1}{K T}\left(E_{F}-E_{c}\right)\right)
$$

while inside the wells,

$$
\begin{aligned}
& W_{2}=W_{c 2} \sum_{i=0}^{L-1}\left|\xi_{i}(y)\right|^{2}( F_{1}\left(\frac{1}{K T}\left(E_{F}-\lambda_{i}\right)\right) \\
&\left.-\frac{1}{k T}\left(E_{c}-\lambda_{i}\right) \ln \left(1+e^{(1 / k T)\left(E_{F}-\lambda_{i}\right)}\right)\right) \\
& W_{3}=\frac{1}{2} \pi\left(\frac{8 m}{h^{2}}\right)^{3 / 2} \int_{\lambda_{L-1}}^{\infty} \frac{\left(E-E_{c}\right)^{3 / 2}}{1+\exp \left((1 / k T)\left(E-E_{F}\right)\right)} d E
\end{aligned}
$$

where $W_{c 2} \equiv\left(4 \pi m k^{2} T^{2} / h^{2}\right)$ and $W_{c 3} \equiv 3(2 \pi m k T /$ $\left.h^{2}\right)^{1.5} k T$.

\section{SOLUTION OF THE EQUATIONS}

The integrals in Eqs. (1.7) and (1.9) are not straightforward Fermi integrals since they have non-zero lower limits, and it is necessary to obtain fast and accurate methods of evaluating these integrals. They may be written in terms of the general integral

$$
I_{r}(a, b) \equiv \frac{1}{\Gamma(r+1)} \int_{b}^{\infty} \frac{z^{r} d z}{1+\exp (z-(a+b))}
$$


Inside the well for example,

$$
n=n_{2}+N_{c 3} I_{(1 / 2)}\left(\frac{1}{k T}\left(E_{F}-\lambda_{L}\right), \frac{1}{k T}\left(\lambda_{L}-E_{c}\right)\right) .
$$

It may be shown [6] that $I_{\mathrm{r}}(\mathrm{a}, \mathrm{b})$ may be written

$$
I_{r}(a, b)=F_{r}(a)+c_{r}(a, b) \ln \left(1+e^{a}\right)
$$

where the function $c_{\mathrm{r}}(\mathrm{a}, \mathrm{b})$ may be approximated by

$$
c_{r}(a, b)=b^{p_{r}}\left(\alpha_{r}+a \beta_{r}\right)
$$

for some values $p_{\mathrm{r}}, \alpha_{r}$ and $\beta_{r}$ which are found by a least mean square search over some suitably defined physical ranges of $a$ and $b$. In the isothermal case previously considered [2]. only $c_{1 / 2}$ was needed, and it was found there that $\alpha_{1 / 2}=$ 0.782 and $\beta_{1 / 2}=-0.023$ when it was assumed that $p_{1 / 2}=0.5$. A more accurate calculation shows that we must take $\alpha_{1 / 2}=0.514 . \beta_{1 / 2}=-0.011$ and $p_{1 / 2}=0.7$. For this new hot electron simulation we must take $\alpha_{3 / 2}=1.420 . \beta_{3 / 2}=0.028$ and $p_{3 / 2}=1.293$.

A rectangular uniform mesh was used on which to discretise the equations. Expressions for $\mathbf{J}$ and $\mathbf{s}$ at the half points were found in terms of the Cfunctions introduced earlier [3]; this method has been shown [4] to be a continuous upwinding method in which the electron temperature at the half point $(i+1 / 2, j)$ has to be written as the logarithmic mean

$$
T_{i+(1 / 2), j}=\frac{T_{i+1, j}-T_{i, j}}{\ln T_{i+1, j}-\ln T_{i, j}} .
$$

At each grid point $(i, j)$ the dependent variables were written as a vector $\mathbf{v}_{i, j} \equiv(\psi, \phi, T)_{i, j}$ and a Newton iteration was used on this vector. The eigenvalues of the Schrödinger equation were found using a QL algorithm with implicit shifts and then ordered. The first $L$ eigenfunctions were then found by back substitution using the Newton method.
The following boundary conditions were applied: $T$ was set equal to the lattice temperature $T_{0}$ on the three contacts, with zero derivatives normal to the free surfaces. On the source and drain, $\psi$ was put equal to the applied voltages $V_{s}$ and $V_{d}$ respectively, while on the gate it was put equal to $V_{g}+\phi_{b}$ where $\phi_{b}$ is the built-in potential. Its normal derivative along the free surfaces along the contact edge was given an artificial slope $\partial \psi_{a}$ in order to pin $E_{c}$ up to an acceptable level for the quantum wells to form. Its normal derivatives on the remaining free surfaces were taken as zero. Given the value of $n$ on these surfaces, the corresponding boundary values for $\phi$ were found by inverting Eq. (1.6) since these surfaces lie outside the quantum wells.

\section{SIMULATION RESULTS}

Steady state results are produced for the device shown in Figure 1 at lattice temperature $T_{0}=300 \mathrm{~K}$. The thicknesses of the layers, starting from the contact edge, are $350 \AA, 320 \AA, 20 \AA$, $45 \AA, 125 \AA, 50 \AA, 50 \AA$ and $1000 \AA$. The proportion $u$ of $\mathrm{Al}$ in layers $2,3,4,6,7$ and 8 is 0.24 , and the proportion $v$ of In in layer 5 is 0.22 . Doping densities are $4.0 \times 10^{24} \mathrm{~m}^{-3}, 1.0 \times 10^{20} \mathrm{~m}^{-3}, 2.0 \times$ $10^{25} \mathrm{~m}^{-3}, 1.0 \times 10^{20} \mathrm{~m}^{-3}, 1.0 \times 10^{20} \mathrm{~m}^{-3}, 1.0 \times$ $10^{20} \mathrm{~m}^{-3}, \quad 1.8 \times 10^{24} \mathrm{~m}^{-3}$, and $1.0 \times 10^{20} \mathrm{~m}^{-3}$. The recess depth is $400 \AA$ and the overall length in the $\mathrm{x}$-direction is $18000 \AA$. The built-in voltage $\phi_{b}$ is taken as $-0.7 \mathrm{~V}$. Data from Monte Carlo simulations performed on the steady state were used to provide the dependence of $m^{*}$ and $\tau$ on $\xi$ [3]. Results shown in Figure 3 relate to the case $V_{g s}=-0.5 \mathrm{~V}$ and $V_{d s}=3.0 \mathrm{~V}$. The iterations were terminated when the residuals reached prescribed minimum values. It was found that taking the artificial slope value of $\partial \psi=-5.0 \times$ $10^{7} \mathrm{~V} \mathrm{~m}^{-1}$ produced a rapidly-forming quantum well profile. The factor $\alpha$ in the Newton upgrade step $\mathbf{v}_{i, j}^{k+1}=\mathbf{v}_{i, j}^{k}+\alpha \delta \mathbf{v}_{i, j}^{k}$ was increased automatically as the iterations progressed. It was started at a very low value and increased to a value not 
greater than 0.2 - a larger value produced an unstable solution. The calculation of the eigensolutions of the Schrödinger equation is computationally very expensive, and it was found that the simulation time could be reduced by iterating to convergence with only three eigensolutions $(L=2)$ and then performing extra iterations using ten eigensolutions $(L=9)$.

\section{References}

[1] Cole, E. A. B., Snowden, C. M. and Boettcher, T. (1997) Int. J. Num. Modelling: Electronic Networks, Devices and Fields, 10, 121.

[2] Cole, E. A. B., Boettcher, T. and Snowden, C. M. (1998). VLSI Design, 8, 29.

[3] Cole, E. A. B. and Snowden, C. M. (1995). Int. J. Num. Modelling: Electronic Networks, Devices and Fields, 8, 13.
[4] Cole, E. A. B. and Snowden, C. M. (2000). Math. and Computer Modelling, 31, 15.

[5] Adachi, S. (1985). J. Appl. Phys., 58, R1.

[6] Cole, E. A. B., Boettcher, T. M. and Snowden, C. M. (1997). Semicond. Sci. Technol., 12, 100.

[7] Cole, E. A. B. (1993). In: Compound Semiconductor Device Modelling, Snowden, C. M. and Miles, R. E. Eds., Springer Verlag, New York, p. 1.

[8] Tang, T.-W. (1984). IEEE Trans. Electronic Devices, ED-31, 1912.

[9] Snowden, C. M. and Loret, D. (1987). IEEE Trans. Electronic Devices, ED-34, 212.

[10] Feng, Y.-K. and Hintz, A. (1988). IEEE Trans. Electronic Devices, ED-35, 1419.

[11] McAndrew, C. C., Heasell, E. L. and Singhal, K. (1988). Semicond. Sci. Technol., 3, 758.

[12] Zhou, J.-R. and Ferry, D. K. (1995). VLSI Design, 3, 159.

[13] Ando, Y. and Itoh, T. (1988). IEEE Trans. Electron Devices, 35, 2295.

[14] Ng, S.-H. and Khoie, R. (1991). IEEE Trans. Electron Devices, 38, 852.

[15] Stern, F. (1984). Phys. Rev. B, 30, 840.

[16] von Roos, O. (1983). Phys. Rev. B, 27, 7547. 

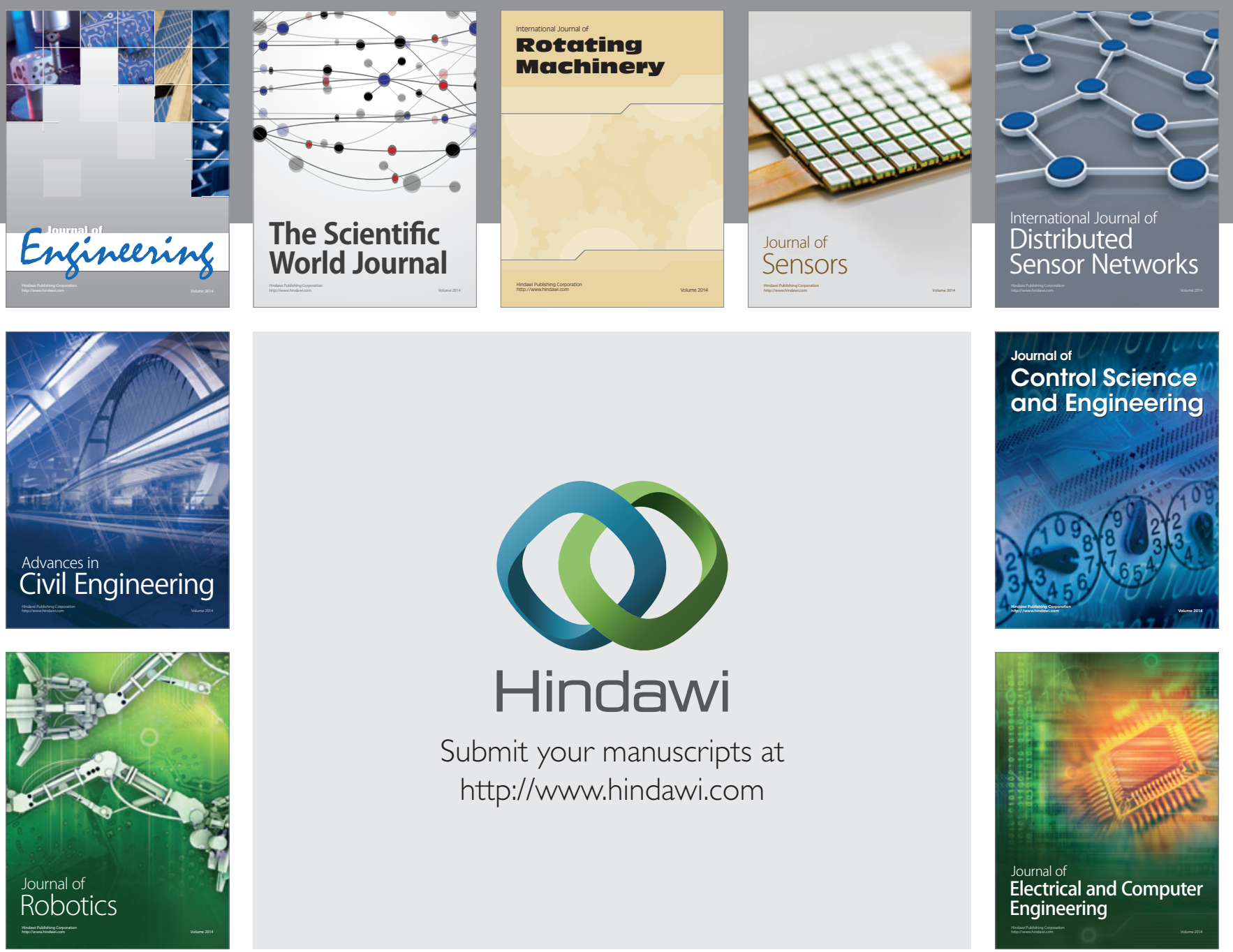

Submit your manuscripts at

http://www.hindawi.com
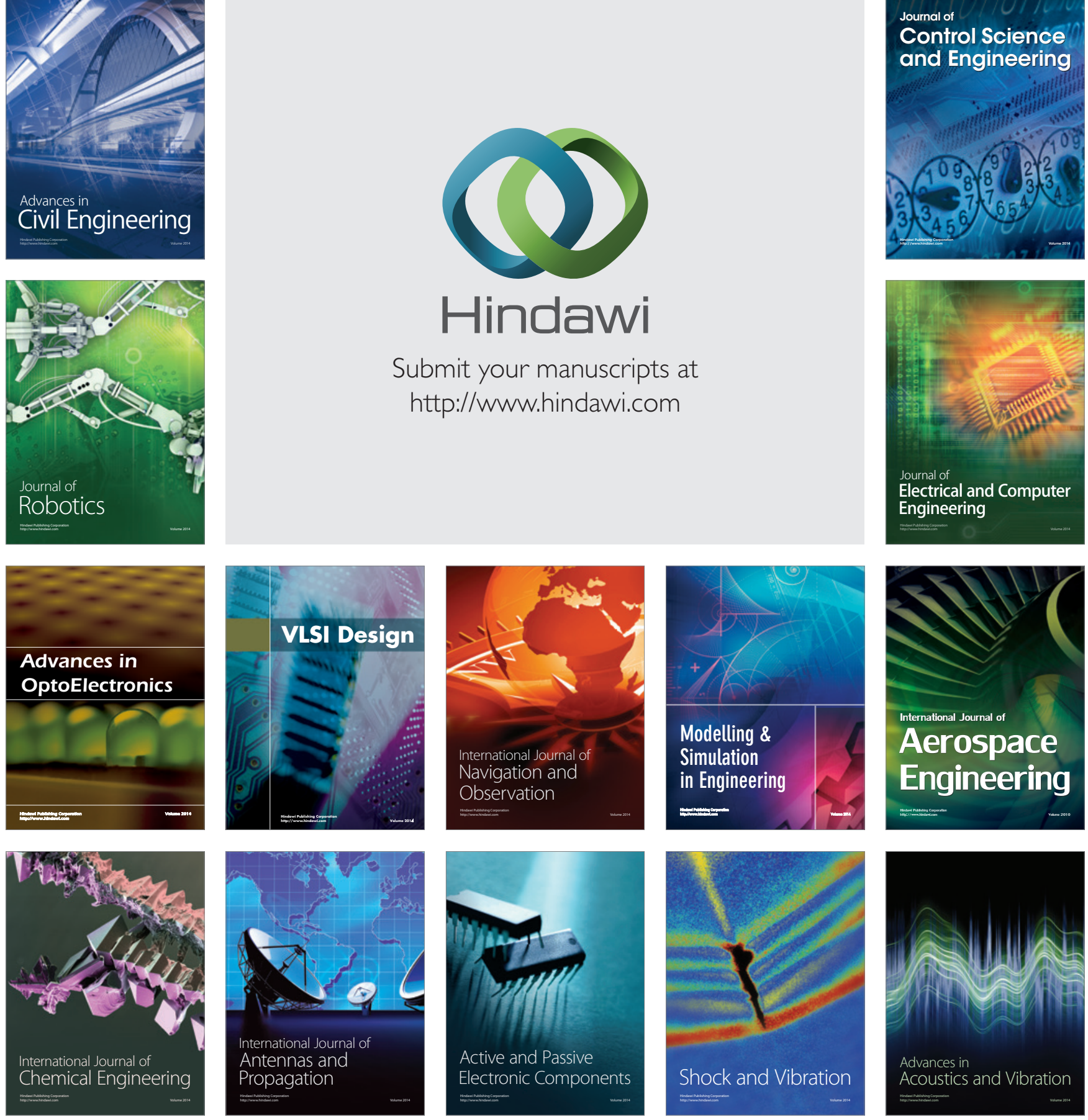